\title{
Anyplace: A Crowdsourced Indoor Information Service
}

\author{
Kyriakos Georgiou*, Timotheos Constambeys*, Christos Laoudias ${ }^{\ddagger}$, \\ Lambros Petrou ${ }^{\S}$, Georgios Chatzimilioudis* and Demetrios Zeinalipour-Yazti* \\ *Department of Computer Science, University of Cyprus, 1678 Nicosia, Cyprus \\ ${ }^{\ddagger}$ KIOS Research Center for Intelligent Systems and Networks, University of Cyprus, 1678 Nicosia, Cyprus \\ ${ }^{\S}$ Department of Computer Science, University of Oxford, OX1 3QD Oxford, UK \\ \{kgeorg10, tconst02\}@cs.ucy.ac.cy; laoudias@ucy.ac.cy; lambros.petrou@wolfson.ox.ac.uk; \{gchatzim, dzeina\}@cs.ucy.ac.cy
}

\begin{abstract}
People do most of their activities, business, commerce, entertainment and socializing indoors. As all of these are increasingly aided by online services and indoor spaces are becoming bigger and more complex, there is a growing need for cost-effective indoor localization, mapping, navigation and information services. In this paper, we present a complete Indoor Information Service, coined Anyplace ${ }^{1}$, which has an open, modular, extensible and scalable architecture, making it ideal for a wide range of applications. Our service features three highly desirable properties, namely crowdsourcing, scalability and accuracy. Anyplace implements a set of crowdsourcing-supportive mechanisms to handle the enormous amount of crowd-sensed data, filter incorrect user contributions and exploit Wi-Fi data from heterogeneous mobile devices. Moreover, it uses a big-data architecture for efficient storage and retrieval of localization and mapping data. Finally, our service relies on the abundance of sensory data on smartphones (e.g., Wi-Fi signal strength and inertial measurements) to deliver reliable indoor geolocation information that received several international awards.
\end{abstract}

Keywords-crowdsourcing, indoor, search, navigation

\section{INTRODUCTION}

People spend $80-90 \%$ of their time in indoor environments ${ }^{2}$, including shopping malls, libraries, airports, university campuses, schools, offices, factories or hospitals. Given that humans are nowadays equipped with mobile computing devices creates a growing need to enterprises, to seek for efficient and cost-effective technology to perform a variety of compelling applications in indoor spaces [2], e.g., workforce tracking, inventory management, supply chain management, security and safety regulations. Retailers are also interested in enhancing shopping experience, analyzing shopping behavior, and offering coupons or advertisements more effectively. Moreover, the entertainment industry envisions to design new games, which could exploit the actual environment of the players as the playground, and communication companies want to offer new services to their customers [2].

Today's technological market and gadget culture allow for the realization of such indoor services with the omni-presence of sensor-rich mobile devices and Wi-Fi Access Points (APs) in indoor environments. Mobile phones can measure the signal strength received from surrounding APs and receive information on their location based on geolocation databases, which

\footnotetext{
${ }^{1}$ Available at: http://anyplace.cs.ucy.ac.cy/

${ }^{2}$ US Environmental Protection Agency, http://epa.gov/iaq/
}

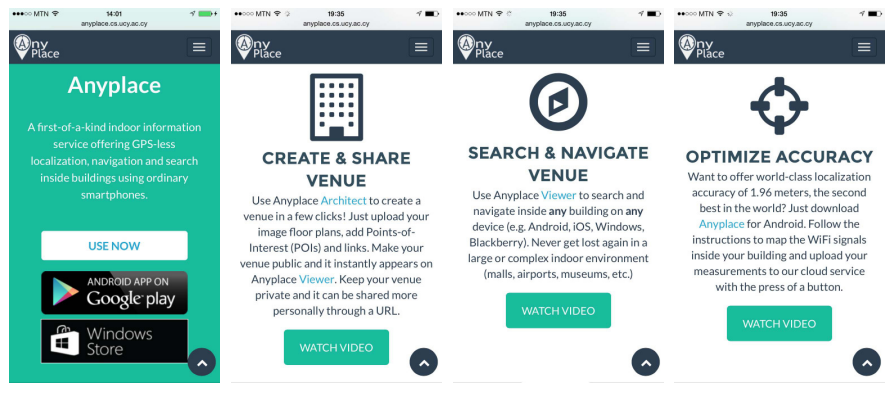

Fig. 1. The Anyplace welcoming and descriptive screens of its features.

maintain information about those APs (i.e., the signal intensity of these transmitters at known locations in space). Fine-grained indoor mapping with detailed Points-Of-Interest (POI) registration can be achieved through crowdsourcing [1], by mobile custodians, of both indoor models and signals. Navigation is then the main extension of all the above, completing what we would call Indoor Information Services (IIS).

Existing IIS services (e.g., SkyhookWireless.com, Google.com, Navizon.com, Infsoft.com, Indoo.rs, IndoorAtlas.com, MazeMap.com) can be categorized based on the localization technology, back-end architecture and participation they use, and on the accuracy and location privacy they achieve. The service can run either on the mobile phone itself (terminal-based) or on a remote server (network-based). The majority of the network-based systems do not provide location privacy to the users, as the localization process is performed on the server that provides location at a coarse granularity (i.e., $\mathrm{km}$ or hundreds of meters) up to a fine granularity (i.e., 1-2 meters) [5]. On the other hand, the terminal-based systems inherently guarantee location privacy as the localization is performed on the mobile device.

Another important distinction among IIS is whether the knowledge base is populated by the service provider alone or by the users in a participatory fashion (crowdsourcing). The commercial systems mostly follow the non-participatory path to populate their databases, which makes them more trustworthy on the data they provide, but forces them to limit their services to the building they were able to map using experts or agents. Systems mainly developed as research projects in academia, follow the participatory scheme, which allows for faster and cheaper database population and maintenance. 


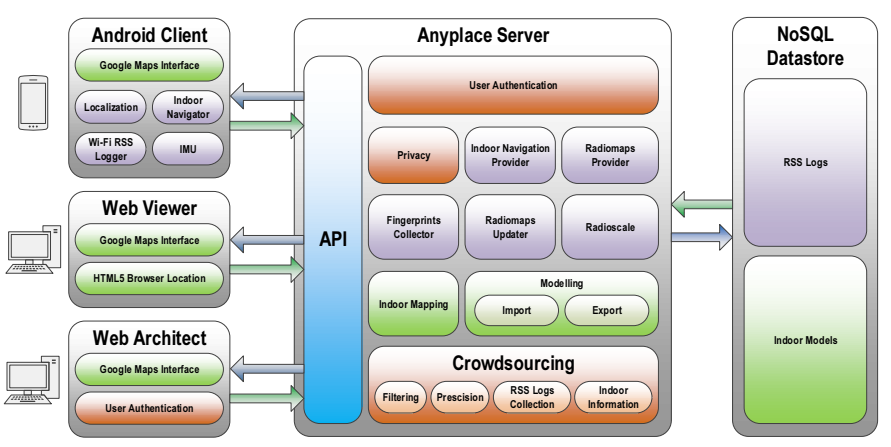

Fig. 2. The Anyplace Indoor Information Service Architecture.

In this article, we present a complete Indoor Information Service, coined Anyplace (an earlier version of our architecture appeared in [4]). Anyplace has an open, modular, scalable and extensible architecture that collects indoor information using crowdsourcing in a user-friendly manner. Anyplace combines the accuracy of a network-based service, and the availability and privacy of a terminal-based service.

The goal of Anyplace is to enable entities, such as individual users, companies, organizations, etc., to realize indoor information management systems, including POI search and navigation, on top of existing wireless networks. It allows for any user to map a building and add POIs, and keeps multiple versions according to the different input data received from different users. For instance, the same convention center would be used one way for one event and in a totally different way, e.g., different POIs for another event. Anyplace implements a set of Map-Reduce mechanisms to handle the enormous amount of crowdsourced sensory data, filter incorrect user contributions and exploit readings collected by heterogeneous mobile devices [3].

We follow a modular architecture that allows to plug-nplay additional modules, either for extending system capabilities by implementing new features (e.g., activity recognition, indoor/outdoor transition), or for enhancing user-experience by improving existing functionalities (e.g., map-matching and sophisticated data fusion to increase localization accuracy). Regarding scalability, Anyplace operates on top of a bigdata management back-end service, with a Google Maps user interface, to ensure efficient and scalable storage and retrieval of localization and mapping data. It also supports offline analytic processing of sensor data using Map-Reduce.

\section{Anyplace System Architecture}

Our platform consists of five main components, including the Server, the Architect, the Viewer, a NoSQL datastore, and a client application running on Android smartphones acting as a Logger and a Navigator (a navigator is also available for Windows Phone). The Anyplace reference system and module architecture is illustrated in Fig. 2.

\section{A. Anyplace Server and Datastore}

The Server follows a big-data architecture and provides a Web 2.0 API using JSON objects for mapping, navigation and localization. It uses Couchbase as its back-end database for scalability and fast metadata retrieval.

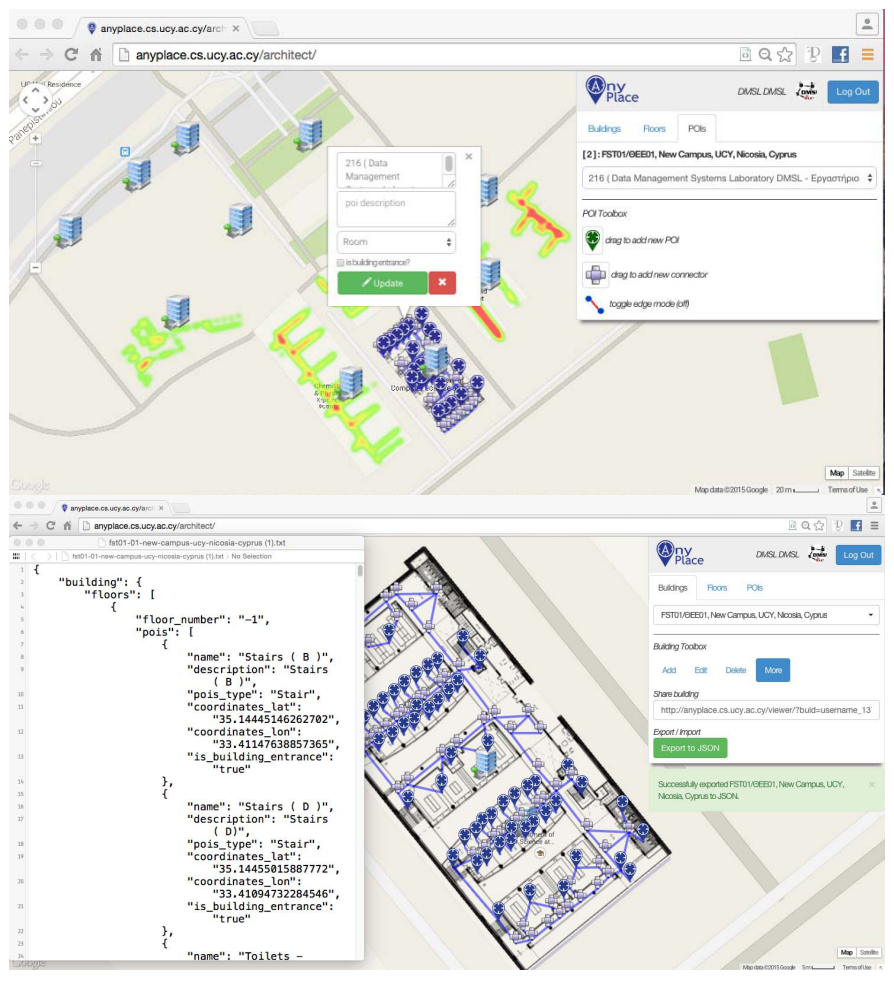

Fig. 3. Anyplace Architect: (Top) Managing a campus of buildings through the architect Web app (cross-platform HTML5 interface). The architect allows a user to add floormaps, POIs and connectors using drag-n-drop. It also allows to overview the collection of Wi-Fi Radiomaps and the management of crowdsourcing tasks. (Bottom) Export/Import of indoor semantics (in JSON) is expected to boost openness and data portability among different indoor management platforms, facilitate data entry and extensibility.

The Server delivers indoor navigation directions to the user upon request based on the Radiomaps stored. The Privacy module enables smartphone users to calculate their location on their own device by downloading minimal parts of the stored Radiomap, "hiding" among $k$ random AP areas. It stores buildings, floorplan maps, and POIs information in JSON, which can be imported and exported. To keep the size of the Radiomap low, the Radio-scale module aggregates all the uploaded RSS values into a grid of predefined resolution.

In addition, the Server features several modules that perform crowdsourcing functionality. Importantly, the differential RSS module outputs RSS differences, instead of absolute RSS values, for fusing the data contributed from devices of various vendors into a single generic Radiomap. In addition, the outlier filtering module checks the validity of the contributed RSS data by comparing the MAC addresses of the APs at the presumed user location to the relevant data in the Radiomap[3].

\section{B. Anyplace Architect}

The Architect Web application offers a feature-rich, userfriendly and account-based interface for managing indoor models in Anyplace (currently a user logs in with any Google account). Particularly, after logging in a user can place the blueprint of a building on top of Google Maps with multifloor support. Using the floor editor, the user can upload, scale and rotate the desired blueprints to fit them properly, as 

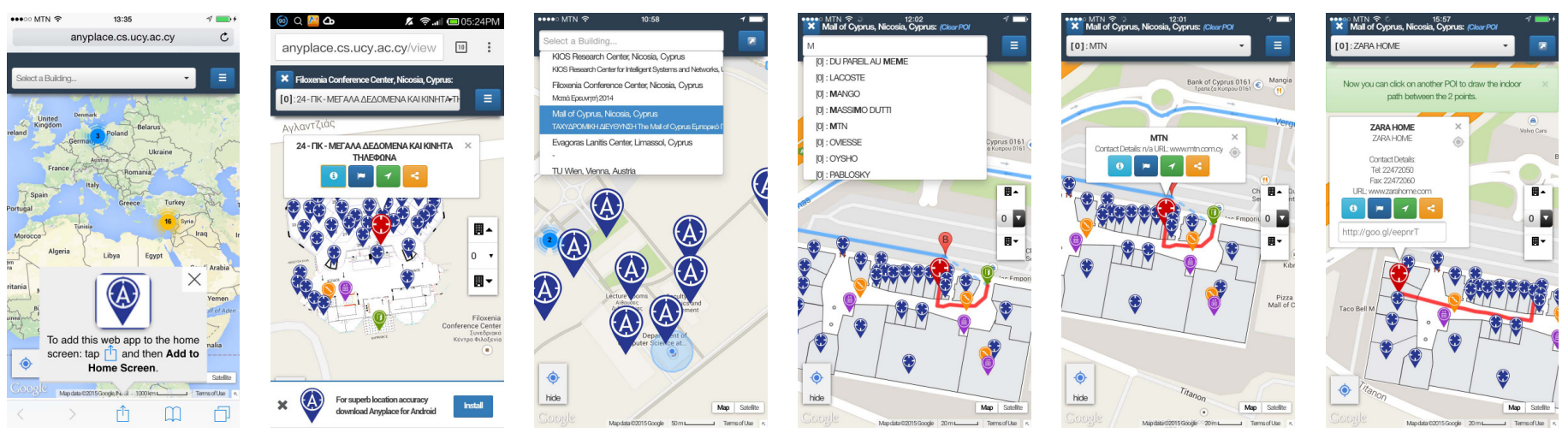

Fig. 4. Anyplace Viewer: (i-ii) Opening Viewer on iOS (i) and Android (ii) presents a clustered-view of Anyplace buildings but also allows users to install the given web app on any smartphone home screen (iOS, Android, Windows, etc.); For Android there is an additional Navigator \& Logger App available through the Play Market; (iii) Location-based Outdoor Search for buildings publicly listed in Anyplace; (iv) Indoor Search of POIs and descriptions belonging to a building selected in a previous step; (v) Oudoor-to-Indoor navigation (outdoor navigation using the Google Maps API); and (vi) Indoor-to-Indoor navigation also showing "URL Sharing" and "Info" options.

shown in Fig. 3. The user can later add, annotate and geo-tag POIs inside the building and connect them to indicate feasible paths for enabling the delivery of navigation directions. This interaction is carried out with drag-n-drop functionality that is cross-browser compatible and even operational on tablets and smartphones used in field deployments (e.g., while moving around in space with a mobile device and correcting the indoor models).

The architect also provides a range of other functionality, namely: (i) invitation/revocation of crowdsourcer accounts to collect Wi-Fi Radiomaps as well as the monitoring of the progress in terms Wi-Fi Radio heat-maps (see Fig. 3 top). An assigner can easily identify whether a given collection is satisfactory or not and thus define quantitative acceptance criteria for the output of crowdsourcers; (ii) making a building public or private, which automatically shares a building on the Anyplace Viewer interface (given that there are no collisions). Alternatively, a building can remain private and be shared among users through a URL (e.g., a person mapping a building for a specific event publicizes a private building to its audience by email or social media); and (iii) export and import of indoor models and Radiomaps, which allows somebody to backup/restore a building, expedite user input of POIs, but also create a new model for a different purpose (e.g., an expo building has different but similar models for different events).

The Architect is a Web App (HTML5, CSS3, JS) that enables the quick visualization of buildings modeled in Anyplace. It's built with the AngularJS framework and it utilizes the Google API (Maps, Directions, Heat-maps and URL shortener) to present and process data on a map along with the HTML5 Geolocation for localization. For the styling of the user interface, the open-source toolkits Bootstrap and FontAwesome were used along with the jQuery UI library that orchestrates user interface interactions and effects.

In the future we aim to: (i) incorporate advanced graph automation tasks, which will check the indoor model (e.g., graph connectivity, automatically connect overlapping stairs and elevators); (ii) provide specific indoor libraries, which will contain different POI types and images for stadiums, malls, campuses, hospitals, etc.; (iii) incorporate algorithms to handle click-streams of error reports and dispute resolution techniques (e.g., using majority voting algorithms); and (iv) investigate the integration of Indoor $G M L^{3}$, which is expected to boost openness and data portability among different indoor management platforms but also facilitate easier data entry.

\section{Anyplace Viewer}

The Viewer is again a Web App that enables the quick visualization of buildings modeled in Anyplace. To appreciate the necessity of this design, assume that a mobile user is lost in some complex University campus. Fortunately, the person sees somewhere an advertisement of the Anyplace service. Upon visiting the website through his mobile internet plan, the user instantly obtains a searchable map of all buildings in the area, performs location-based range search and quickly gets to his target. The UX/UI of the Viewer has been implemented with a mobile user on-the-go in mind (i.e., thumb-based user interface, large buttons, less clutter) and is extremely straightforward to use.

The Viewer is ideal for a first-time user that doesn't want to waste considerable time before launching the service through an app downloaded from some mobile market. The viewer enables off-the-shelf usage without installation or logistical challenges, which is many times an overhead when users aim to get to their destination quickly. A more involved user can download the Anyplace Navigator from the various markets and enjoy advanced functionality (e.g., superb accuracy, performance, caching and prefetching, privacy, etc.)

One final note is that the Viewer enables a user to share short URLs of POIs using email, the web, social media, etc. A user receiving such a URL can instantly project navigation instructions to the target POI. This functionality has already been extensively incorporated in various websites in the form of a Viewer Widget (i.e., the viewer is installed and executed within any external website using iframes). The Viewer has been implemented on a common codebase with the Architect.

\section{Anyplace Navigator \& Logger}

The combined Navigator and Logger is a designated tool for Android users, which can benefit from Wi-Fi fingerprinting [4], [5] available under this platform. The Navigator allows

\footnotetext{
${ }^{3}$ IndoorGML, http://indoorgml.net/
} 

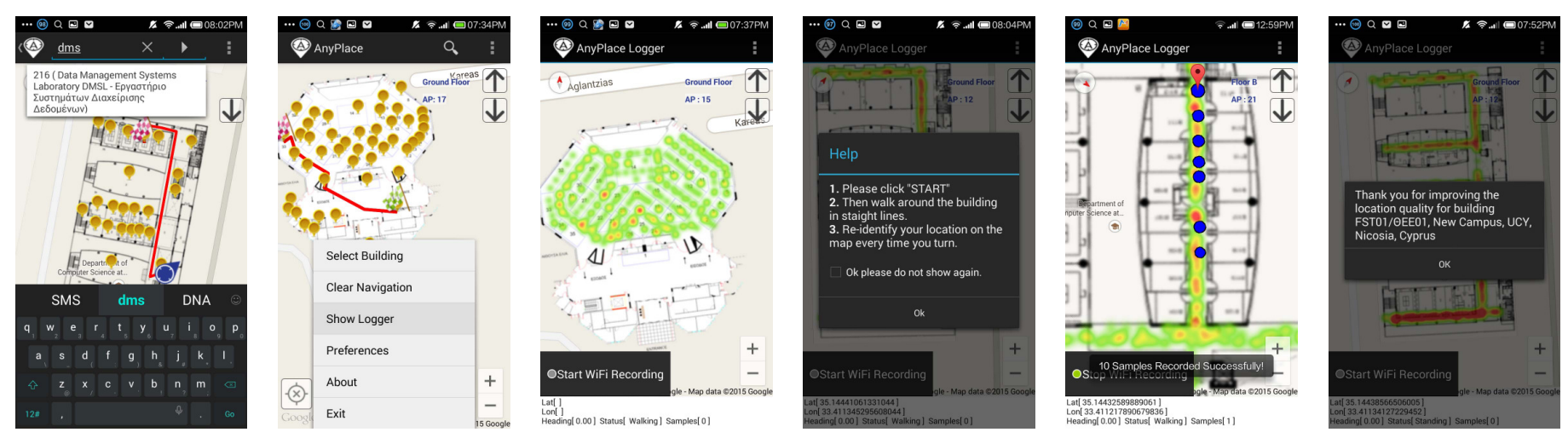

Fig. 5. Anyplace Navigator and Logger: (i) Search and Navigate: A user executing Navigator searches for POIs and receives navigation instructions using accurate local IMU and Wi-Fi algorithms; (ii) Open Logger: While browsing another distant building, the user opens the Logger; (iii) Heat-map: The user now knows where to initiate the collection of Wi-Fi fingerprints given that certain areas are not covered (i.e., here the logging will fail as the user is not inside the given building); (iv) Guide: The user returns to the building he is currently at and receives instruction on how to improve the Radiomap of the venue; (v) Collect: The user walks in straight lines and locally collects the data; and (vi) Upload: The user uploads the Wi-Fi signals to the Anyplace cloud service.

users to see their current location on top of the floorplan map and navigate between POIs inside the building, similarly to the Viewer. The main difference, is that the Navigator now offers superb accuracy of 1.96 meters, which received the second award by Microsoft Research at IEEE IPSN'14 [5]. The Logger application enables users to record RSS readings from nearby Wi-Fi APs and upload them to our Server through a Web 2.0 API (in JSON).

A user installing Anyplace from the Google Play market can use the Navigator in any building listed on Anyplace (public or private). The same applies to the Logger, with the difference that Wi-Fi logging can only be enabled if a user is actually inside a given building (similarly to waze.com). This allows the Anyplace service to minimize the reception of wrong signals from crowdsourcers, which will only be able to contribute data if they are physically inside a building.

The Navigator also uses the onboard smartphone sensors (i.e., accelerometer, gyroscope and digital compass), which are seamlessly integrated in our tracking module to smooth the Wi-Fi locations and enhance the navigation experience. When the Navigator is launched, the building map and the associated POIs are automatically loaded by using the rough user location provided by the Google Geolocation API (Fig. 5). Then, the application downloads the RSS Radiomap of the relevant floor (subsequently the complete building) and displays the user location on top of the map. Moreover, users may search for POIs and get navigation directions from their current location.

The Logger on the other hand is used by volunteers for contributing RSS data and for crowdsourcing the Radiomap. Crowdsourcers can select the desired building and floor, modify the walking sensitivity as well as other settings, through the preferences screen. Subsequently, the users indicate their current location by clicking on the map and then click the on-screen buttons to initiate and end the logging process. In order to facilitate the collection of quality Radiomaps, we present a heat-map of previously collected fingerprints in the building. A crowdsourcer seeing this heat-map can easily identify areas where additional samples have to be collected. If a crowdsourcer is outside a given building no Wi-Fi signals can be collected. Upon finishing the collection of data, a user can upload this data to the Anyplace cloud service.

\section{DEMONSTRATION EXPERIENCE}

Our system has low setup time because it will rely on the Wi-Fi infrastructure available at the conference venue. If necessary, our team can quickly deploy additional smartphone APs to provide adequate Wi-Fi coverage. Thus, only the floorplan digital map of the demo site is required.

We will demonstrate the real-time localization capabilities of Anyplace by allowing attendees to carry an Android smartphone running the Navigator software and viewing their travelled path, while walking around the demo area. At the same time the participants will be able to appreciate the potential of indoor location-oriented services by carrying out POI search and navigation.

They will also have the opportunity to interact with the Architect website in order to add and annotate indoor spaces in a straightforward way or simply explore other annotated buildings that are publicly available through the Viewer mode.

\section{ACKNOWLEDGMENTS}

This work was supported by the Univ. of Cyprus (Startup Grant / Zeinalipour), by MTN Cyprus, EU's COST Action IC903 and IC1304, EU's FP7 MODAP project.

\section{REFERENCES}

[1] G. Chatzimilioudis, A. Konstantinidis, C. Laoudias, and D. Zeinalipour-Yazti, "Crowdsourcing with smartphones," IEEE Internet Computing, vol. 16, no. 5, pp. 36-44, 2012.

[2] D. Dodge, "Why Indoor Location will be bigger than GPS or Maps, and how it works", Online: http://goo.gl/jOvGqn, Apr 23, 2014.

[3] C. Laoudias, D. Zeinalipour-Yazti, and C.G. Panayiotou, Crowdsourced indoor localization for diverse devices through radiomap fusion, Indoor Positioning and Indoor Navigation (IPIN), 2013 International Conference on, Oct 2013, pp. 1-7.

[4] L. Petrou, G. Larkou, C. Laoudias, D. Zeinalipour-Yazti, and C. G. Panayiotou, "Crowdsourced indoor localization and navigation with anyplace," in Proceedings of the 13th Intl. Conference on Information Processing in Sensor Networks, pp. 331-332, 2014.

[5] D. Lymberopoulos, J. Liu, X. Yang, R. R. Choudhury, ..., C. Laoudias, D. Zeinalipour-Yazti, Y.-K. Tsai, and et. al., "A realistic evaluation and comparison of indoor location technologies: Experiences and lessons learned," in Proceedings of the 14th IEEE/ACM International Symposium on Information Processing in Sensor Networks, 2015. 